\title{
Effect of hydroxyapatite-containing microspheres embedded into three-dimensional magnesium phosphate scaffolds on the controlled release of lysozyme and in vitro biodegradation
}

\author{
This article was published in the following Dove Press journal: \\ International Journal of Nanomedicine \\ I September 2014 \\ Number of times this article has been viewed
}

Jongman Lee

Hui-suk Yun

Powder and Ceramics Division, Korea Institute of Materials Science, Changwon, Republic of Korea
Correspondence: Hui-suk Yun Powder and Ceramics Division, Korea Institute of Materials Science (KIMS), 797 Changwondaero, Seongsangu, Changwon 642-83I, Republic of Korea

$\mathrm{Tel}+8255280335$ I

Fax +82552803392

Email yuni@kims.re.kr
Abstract: The functionality of porous three-dimensional (3D) magnesium phosphate (MgP) scaffold was investigated for the development of a novel protein delivery system and biomimetic bone tissue engineering scaffold. This enhancement can be achieved by incorporation of hydroxyapatite (HA)-containing polymeric microspheres (MSs) into a bulk $\mathrm{MgP}$ matrix, and a paste-extruding deposition (PED) system. In this work, the amount of MS and HA was precisely controlled when manufacturing MS-embedded $\mathrm{MgP}(\mathrm{MS} / \mathrm{MgP})$ composite scaffolds. The main influence was researched in terms of in vitro lysozyme-release, in vitro biodegradation, mechanical properties, and in vitro calcification. The controlled release of lysozyme was indicated, while showing graded release patterns according to HA content. The composite scaffolds degraded gradually with MS content and degradation time. Due to the effect of HA inclusion, the higher HA-containing MS/MgP scaffolds could, not only delay the biodegradation process but also, compensate for the possible loss of mechanical properties. In this regard, it is reasonable to confirm the inverse relationship between biodegradation and corresponding compressive properties. In order to encourage bioactivity and osteoconductivity, the MS/MgP composite scaffolds were subjected to simulated body fluid treatment. Calcium deposition was, in turn, improved with increasing MS and HA content over time. This quantitative result was also proved using morphological and elemental analysis. In summary, a significant transformation of a monolithic MgP scaffold was directed toward a multifunctional bone tissue engineering scaffold equipped with controlled protein delivery, biodegradability, and bioactivity.

Keywords: protein delivery, bone tissue engineering

\section{Introduction}

The usefulness of microspheres (MSs) has been extended to combination with a continuous bulk matrix: solid polymer, hydrogel, and calcium phosphate cement (CPC). ${ }^{1}$ To this end, they can affect physicochemical and biological characteristics, in terms of porosity, ${ }^{2}$ mechanical properties, ${ }^{2}$ biodegradation, ${ }^{3}$ the release kinetics of bioactive agents, ${ }^{4}$ and bioactivity. ${ }^{3}$ One of the greatest advantages of introducing MS into a bulk matrix is to allow final composite scaffolds controlled delivery of biomolecules in a spatiotemporal manner. This is mainly because the direct incorporation of bioactive agents into bulk scaffolds might be vulnerable to denaturation when exposed to harsh preparation conditions (organic solvent, acidic byproduct, hydrophobicity, and so on). ${ }^{5}$

First, a composite system of MS-embedding solid polymers was developed for the sustained release of biomolecules and promoted tissue regeneration compared with 
MS-free scaffolds. ${ }^{3,6}$ Porous nano-hydroxyapatite (HA)/ collagen/poly(L-lactic acid) (PLLA) composite scaffolds containing chitosan MSs were prepared using a thermally induced phase separation method. ${ }^{3}$ The compressive properties increased as the chitosan MS content increased, but the porosity decreased. The in vitro biodegradation rate increased with the enhancement of chitosan MSs, which in turn led to temporally controlled release of synthetic peptide. Second, a composite system of MS-embedding hydrogel was devised to serve as a controlled drug delivery carrier, ${ }^{4}$ reinforcement component, ${ }^{7}$ and cell delivery vehicle. ${ }^{8}$ The biodegradable gelatin MS/hydrogel composites were prepared to accomplish a simultaneous delivery of transforming growth factor- $\beta 1$ (TGF- $\beta 1$ ) and chondrocytes, respectively, for cartilage tissue engineering applications. ${ }^{4}$ As a result, a much slower release of TGF- $\beta 1$ to the gelatin MS-encapsulating hydrogel composites was seen over the course of 28 days. Injectable alginate hydrogel composites combined with $\beta$-tricalcium phosphate ( $\beta$-TCP) MSs were invented to overcome the lack of initial mechanical strength of the hydrogel constructs for bone tissue engineering. ${ }^{7}$ Third, a composite system of MS-embedding CPC was researched extensively to solve the major drawbacks of CPC: slow degradation rate attributed to a lack of macroporosity and poor drug-release capacity. ${ }^{1}$ In this regard, many types of biodegradable polymeric MSs were homogeneously incorporated into bone cements, not only to facilitate in vitro/in vivo biodegradation and the resultant formation of well-interconnected macroporous structures, but also, to obtain the controlled release of bioactive agents for a prolonged period of time, without an initial drug burst. ${ }^{9-11}$

In our previous studies, we developed a novel roomtemperature process of three-dimensional (3D) magnesium phosphate $(\mathrm{MgP})$ scaffold fabrication, using a paste-extruding deposition (PED) system. ${ }^{12}$ This technique enabled us to directly blend lysozyme, as a model bioactive substance, into $\mathrm{MgP}$ powder, to introduce homogeneous distribution in the scaffold. This was completely ascribed to a simple cementation process at room temperature, instead of the typical sintering process at high temperature. Both the drugloading efficiency and in vitro release performance were, in turn, substantially enhanced compared with physical adsorption using lysozyme-containing solution. Nevertheless, we wished to develop a more advanced delivery system with a high efficacy. We recently investigated the manufacture of HA-containing polymeric MSs as a novel protein delivery carrier. ${ }^{13}$ High protein-loading capacity and controlled release kinetics were achieved by strong electrostatic interactions between nanosized HA and the lysozyme protein. For this reason, we combined the $\mathrm{MgP}$ scaffold with HA-containing polymeric MSs to produce the $3 \mathrm{D}$ bone tissue engineering composite scaffold.

In the present work, our aim was to develop MSembedding MgP (MS/MgP) composite scaffolds that could contribute to in vitro lysozyme-release characteristics, in vitro biodegradation/corresponding mechanical properties, and in vitro calcification, in contrast to the bare MgP scaffold. For this purpose, MS/MgP composite scaffolds were prepared into two groups: (1) controlled MS content in $\mathrm{MS} / \mathrm{MgP}$ scaffolds, ranging from $0 \%$ to $20 \%(\mathrm{w} / \mathrm{w})$, and (2) controlled HA content in MSs, ranging from $0 \%$ to $55 \%$. A synergistic combination of MgP scaffold and HAcontaining polymeric MSs will be useful to create an efficient protein delivery carrier and bioactive physical platform for bone tissue engineering.

\section{Materials and methods Preparation of MSs and MgP powder}

The development of gelatin/chitosan MSs, consisting of nanosized HA (Sigma-Aldrich Corp., St Louis, MO, USA), was reported in our previous publication. ${ }^{13}$ Briefly, $2.5 \%$ gelatin type B/chitosan with a weight ratio of 2 to 1 (SigmaAldrich Corp.) was dissolved in $50 \mathrm{~mL}$ of $0.1 \mathrm{M}$ acetic acid (Sigma-Aldrich Corp.). Different amounts of HA ( $0 \%, 1.5 \%$, and $3.0 \%[\mathrm{w} / \mathrm{v}]$ ) were added to this solution and ultrasonicated for 5 minutes, for homogeneous dispersion of the HA. MSs with no HA were made for comparison following the same protocol. In this work, the theoretical percentage of HA in the total solid content was $38 \mathrm{wt} \%$ for a mixture containing $1.5 \%(\mathrm{w} / \mathrm{v}) \mathrm{HA}$ and was $55 \mathrm{wt} \%$ for $3.0 \%$ (w/v) HA. For this reason, the final composite MSs with different HA content $(0 \%, 38 \%$, and $55 \%)$ were denoted as MS ( $0 \%$ HA), MS (38\% HA), and MS (55\% HA), respectively. The mixture was added dropwise into 5\% cellulose acetate butyrate (Eastman Chemical Co., Kingsport, TN, USA) dissolved in $200 \mathrm{~mL}$ of n-butyl acetate (Samchun Pure Chemical Co., Ltd., Seoul, Republic of Korea) while stirring at $200 \mathrm{rpm}$ for 30 minutes. The gelatin/chitosan MSs were subsequently cross-linked by adding glutaraldehyde (Merck \& Co., Inc., Whitehouse Station, NJ, USA) to the emulsion at a final concentration of $0.25 \%$, and stirred overnight. The composite MSs were then washed with acetone, rinsed in deionized (DI) water, and then collected using sieves with $150 \mu \mathrm{m}$ and $250 \mu \mathrm{m}$ pores. The residual glutaraldehyde from the cross-linking process was neutralized by placing the MSs in $2.0 \%$ sodium bisulfide for 1 day and then washing with DI water. The washed MSs 
were lyophilized for 1 day and stored in a desiccator until further use.

$\mathrm{MgP}$ powder (farringtonite $\left[\mathrm{Mg}_{3}\left(\mathrm{PO}_{4}\right)_{2}\right]$ ) was prepared following the methods described in our previous publication. ${ }^{12}$ Briefly, $\mathrm{Mg}(\mathrm{OH})_{2}$ (Junsei Chemical Co., Ltd., Tokyo, Japan) and $\mathrm{H}_{3} \mathrm{PO}_{4}$ (DC Chemical Co., Ltd., Seoul, Korea) were reacted with a molar ratio of $3 \mathrm{M}$ to $2 \mathrm{M}$. Then, $2 \mathrm{M}$ of $\mathrm{H}_{3} \mathrm{PO}_{4}$ was added to a $3 \mathrm{M}$ of $\mathrm{Mg}(\mathrm{OH})_{2}$ suspension in a dropwise manner. The solution was mixed uniformly for 10 hours and left for 24 hours to age. The precipitate was filtered and dried at $80^{\circ} \mathrm{C}$ for 2 days and heat-treated at $850^{\circ} \mathrm{C}$ for 6 hours. The final product was ground in a planetary ball mill for 2 hours at a speed of $250 \mathrm{rpm}$.

\section{Preparation of MS/MgP composite scaffold}

In this work, the preparation of MS/MgP composite scaffolds could be categorized into two groups: (1) controlled MS content in MS/MgP scaffolds and (2) controlled HA content in MS. The MS content ranged from $0 \%$ to $20 \%$ (w/w) in the $55 \%$ HA-containing MS/MgP scaffolds; the HA content ranged from $0 \%$ to $55 \%$ in the $10 \% \mathrm{MS} / \mathrm{MgP}$ scaffolds. The HA-containing composite microspheres and $\mathrm{MgP}$ powder were mixed together using a 1\% hydroxypropyl methyl cellulose solution (HPMC) (Sigma-Aldrich Corp.), with a powder/liquid ratio of 3 to 2-3 (wt:vol). A uniform $\mathrm{MS} / \mathrm{MgP}$ green paste was formed for the PED system. In order to visualize the MSs in the MS/MgP composite scaffolds, as shown in Figure 1, MS (55\% HA) was stained with $0.4 \%$ trypan blue solution (Gibco ${ }^{\circledR}$; Life Technologies, Carlsbad, CA, USA), washed in DI water vigorously, and lyophilized for 1 day, prior to mixing with $\mathrm{MgP}$ powder. The $\mathrm{MS} / \mathrm{MgP}$ paste, housed in a syringe, was mounted to a gantry robotic deposition apparatus, and equipped with speciallyaltered systems, such as an actuator, to control the position of the deposition nozzle for the fabrication of 3D structures. A nozzle size of $21 \mathrm{G}$ was used to fabricate the $3 \mathrm{D} \mathrm{MS} / \mathrm{MgP}$ scaffolds $(10 \times 10 \times 4 \mathrm{~mm})$ with a center-to-center distance of $1 \mathrm{~mm}$. The MS/MgP scaffold green bodies were dried at room temperature, followed by immersion in $3.5 \mathrm{M}$ diammonium hydrogen phosphate (DAHP) (Junsei Chemical Co., Ltd.) for 1 day, with gentle shaking to induce a cement reaction. They were then washed in DI water three times and dried completely by lyophilization. The MgP scaffold without the inclusion of MSs was also prepared for comparison. The final MS/MgP scaffolds were observed under a stereomicroscope (SMZ 1,500; Nikon Corp., Tokyo, Japan) to examine the morphology, strut thickness, and pore size $(n=5)$. The apparent porosity of MS/MgP scaffolds (n=5) was calculated according to the following equation:

$$
\text { Porosity }=\left[1-\left(\mathrm{D}_{\text {scaffold }} / \mathrm{D}_{\text {bulk }}\right)\right] \times 100 \text {, }
$$

where $\mathrm{D}_{\text {scaffold }}$ means density of scaffold and $\mathrm{D}_{\text {bulk }}$ is density of bulk.

\section{Morphological analysis of MS/MgP composite scaffold}

For the morphological analysis, MS/MgP composite scaffolds were first sputter-coated with gold to minimize sample charging problems and evaluated by scanning electron microscope (SEM) (JSM-6610LV; JEOL Ltd., Tokyo, Japan) at an operating voltage of $15 \mathrm{kV}$. Surface and cross-sectional SEM micrographs were taken for each MS/MgP composite scaffold, at magnifications of $\times 50$ and $\times 100$.

\section{Water absorption}

For this test, the various MS (55\% HA) content samples, ranging from $0 \%$ to $20 \%(\mathrm{w} / \mathrm{w})$, were selected to constitute $\mathrm{MS} / \mathrm{MgP}$ composite scaffolds. The water absorption of the $\mathrm{MS} / \mathrm{MgP}$ composite scaffolds was determined by measuring hydrated weight in DI water at $37^{\circ} \mathrm{C}$ for 3 hours, with gentle shaking ( $\mathrm{n}=5)$. MS/MgP scaffolds were then carefully patted with tissue to remove excess water and weighed to measure the hydrated weight $\left(\mathrm{W}_{\mathrm{h}}\right)$. The percentage increase in water absorption can be calculated in contrast to the dried weight $\left(\mathrm{W}_{\mathrm{d}}\right)$ of the MS/MgP scaffolds, according to the following equation:

$$
\text { Water absorption }(\%)=\left(\left[\mathrm{W}_{\mathrm{h}}-\mathrm{W}_{\mathrm{d}}\right] / \mathrm{W}_{\mathrm{h}}\right) \times 100
$$

\section{Compressive properties}

Based on the MS/MgP scaffold fabrication method described above, MS/MgP composite scaffolds with different MS- and HA-content were produced, while controlling the strut distance of $1.0 \mathrm{~mm}$ and nozzle size of $21 \mathrm{G}$. The dimension of the $\mathrm{MS} / \mathrm{MgP}$ scaffolds was $10 \times 10 \times 4 \mathrm{~mm}$. The compressive strength and modulus were measured using a uniaxial testing machine, with a $2 \mathrm{kN}$ load cell at a cross-head rate of $1 \mathrm{~mm} / \mathrm{min}$ (RB Model 302 ML $^{\text {тм}}$; R\&B Inc., Daejon, Korea) (n=5).

\section{In vitro biodegradation and compressive properties}

$\mathrm{MS} / \mathrm{MgP}$ composite scaffolds, with a dimension of $10 \times 10 \times$ $4 \mathrm{~mm}$, were measured for dried weight prior to beginning this test. In order to provide the accelerated conditions for an 
A

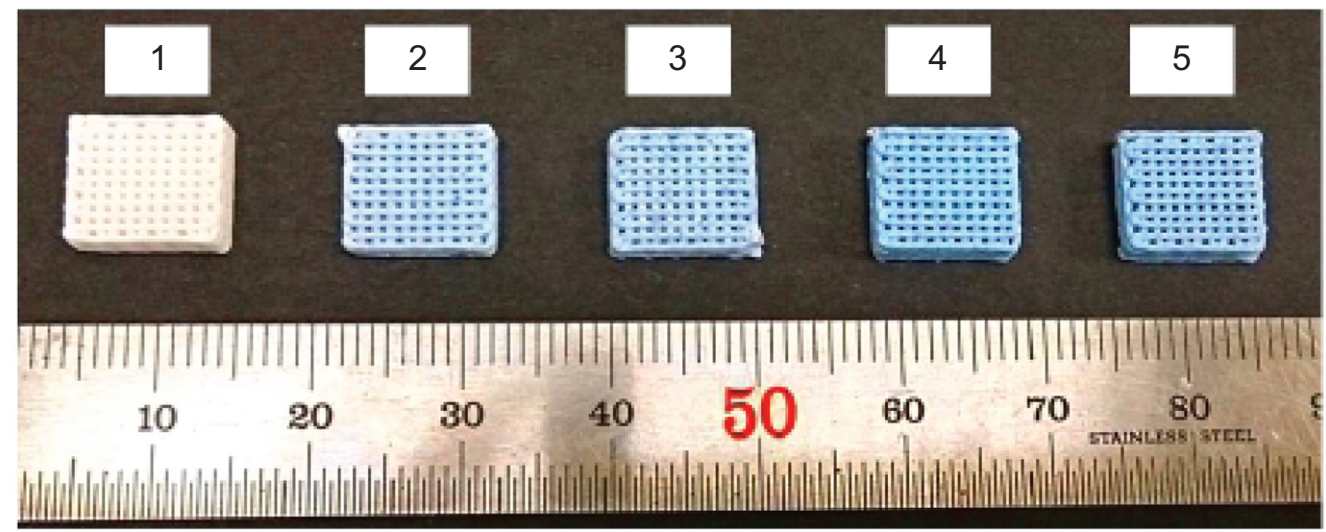

B
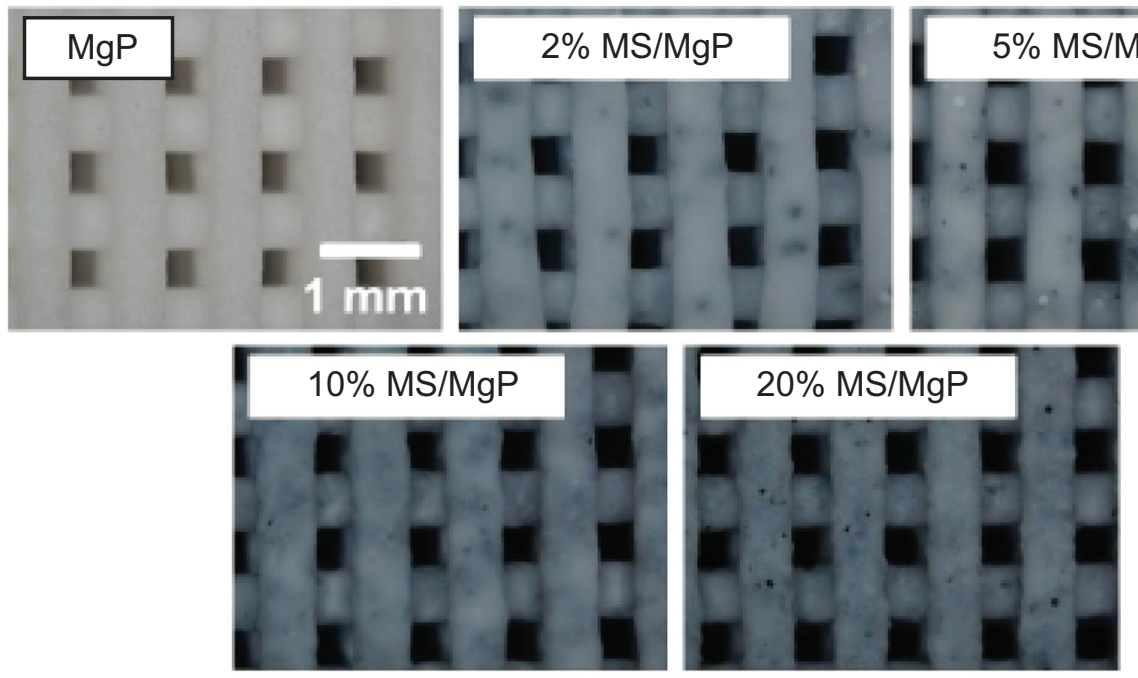

Figure I Gross images of MS/MgP composite scaffolds.

Notes: Gross images of MS/MgP composite scaffolds (A): (I) MgP, (2) 2\% MS/MgP, (3) 5\% MS/MgP, (4) I0\% MS/MgP, and (5) 20\% MS/MgP. Closer observation of MS/MgP composite scaffolds, via a stereomicroscope $(\times 3$ magnification) $(\mathbf{B})$.

Abbreviations: MgP, magnesium phosphate; MS, microsphere.

in vitro biodegradation test, $10 \mathrm{~mL}$ of collagenase solution (50 $\mu \mathrm{g} / \mathrm{mL}$ ) (Sigma-Aldrich Corp.) containing $0.1 \% \mathrm{NaN}_{3}$ (Sigma-Aldrich Corp.) was transferred, to submerge the MS/ MgP scaffolds fully during the whole incubation time. Samples were placed in an incubator temperature-controlled at $37^{\circ} \mathrm{C}$, while providing gentle shaking. At each predetermined time (days 5, 15, and 30), samples were collected, washed with DI water three times, and lyophilized to obtain the dried weight. The percentage of weight reduction was then calculated in comparison with the initial dried weight $(n=5)$. The corresponding compressive properties at each biodegradation time $(n=5)$ were evaluated as well, in terms of compressive strength and modulus, to investigate the relationship between in vitro biodegradation and compressive properties.

\section{In vitro lysozyme-release kinetics}

In order to investigate the release kinetics of lysozyme from the MS/MgP composite scaffolds, 10\% MS with different HA content ( $0 \%$ to $55 \%$ ) was incubated overnight with $2 \mathrm{~mL}$ of lysozyme solution in phosphate-buffered saline (PBS) $(0.5 \mathrm{mg} / \mathrm{mL})$. The supernatant was then collected to measure ultraviolet (UV) absorbance at $280 \mathrm{~nm}$, and the amount of lysozyme loaded into the composite MSs was calculated based on a standard curve, prepared using a series of lysozyme concentrations. The composite MSs were then washed once with DI water to eliminate nonspecifically bound lysozyme and lyophilized, prior to blending with $\mathrm{MgP}$ powder. Subsequently, 10\% $\mathrm{MS} / \mathrm{MgP}$ composite scaffolds were made following the same method shown above. For comparison, a direct addition of lysozyme into $\mathrm{MgP}$ scaffold was also prepared without using MSs as a lysozyme carrier. The amount of lysozyme for this sample was the same as that loaded into MS (55\% HA), which was the largest lysozyme content in all the MS samples with different HA content. Briefly, lysozyme, dissolved in 1\% HPMC solution, was uniformly 
blended with $\mathrm{MgP}$ powder to achieve a direct incorporation of lysozyme inside MgP scaffold. After lyophilizing the lysozyme-loading scaffolds, the dried weight was measured to calculate the lysozyme-loading content per scaffold; $0.37 \mathrm{~g}$ for $\mathrm{MgP}, 0.27 \mathrm{~g}$ for $\mathrm{MS}(0 \% \mathrm{HA}) / \mathrm{MgP}$, $0.32 \mathrm{~g}$ for $\mathrm{MS}(38 \% \mathrm{HA}) / \mathrm{MgP}$, and $0.32 \mathrm{~g}$ for $\mathrm{MS}(55 \%$ $\mathrm{HA}) / \mathrm{MgP}(\mathrm{n}=5)$. The lysozyme-release test began by placing MS/MgP composite MSs into $5 \mathrm{~mL}$ of PBS containing $0.1 \% \mathrm{NaN}_{3}$ at $37^{\circ} \mathrm{C}$, with gentle shaking (150 rpm). At each predetermined time, a sample of the medium was collected and replaced with an equal volume of fresh medium. The lysozyme content was analyzed quantitatively using a micro $\mathrm{BCA}^{\mathrm{TM}}$ protein assay kit (Thermo Fisher Scientific, Inc., Waltham, MA, USA) according to the manufacturer's instructions $(n=5)$.

\section{In vitro calcification}

The capacity of MS/MgP composite scaffolds $(10 \times 10 \times 4$ $\mathrm{mm}$ ) to absorb calcium ions was estimated by adding each $\mathrm{MS} / \mathrm{MgP}$ scaffold to $10 \mathrm{~mL}$ of simulated body fluid (SBF) and incubating at $37^{\circ} \mathrm{C}$ for 5,10 , and 20 days, with gentle shaking. The SBF was prepared according to previously reported studies. ${ }^{14}$ At each predetermined time (day 5, 10, and 20), SBF was collected and replaced with fresh fluid. The calcium ion content deposited on the MS/MgP composite scaffolds was then determined qualitatively using a commercially available assay kit (QuantiChrom ${ }^{\mathrm{TM}}$ Calcium Assay Kit, BioAssay Systems, Hayward, CA, USA) $(n=5)$. The initial calcium ion concentration of SBF was compared with the retrieved SBF solution at each time noted, to calculate the calcium ion deposition. In brief, $5 \mu \mathrm{L}$ of retrieved samples were reacted with $200 \mu \mathrm{L}$ of working solution and incubated for 3 minutes at room temperature, followed by measurement of UV absorbance at $612 \mathrm{~nm}$, using a UV-Visible spectrophotometer (UVmini-1240; Shimadzu Corp., Kyoto, Japan). After completing the calcium ion deposition test in SBF, the samples at day 20 were washed carefully with DI water and dried thoroughly to carry out a morphological and elemental analysis by SEM and energy dispersive spectroscopy (EDS) (Oxford Instruments, Abingdon, UK), respectively.

\section{Statistics}

All quantitative results were expressed as means \pm standard deviation for number $n$ of samples analyzed. Statistical analysis was carried out using one-way analysis of variance (ANOVA), followed by the Tukey test for post hoc multicomparisons to determine whether the differences were significant. A value of $P<0.01$ or $P<0.05$ was accepted as statistically significant.

\section{Results and discussion Microscopic characterization of MS/MgP composite scaffolds}

HA-containing composite MS was prepared according to the preparation method described above. As described in our previous publication, the mean diameters for the composite MS in the dried state increased slightly with increasing HA content, from $85 \pm 11 \mu \mathrm{m}$ to $95 \pm 12 \mu \mathrm{m}$ to $101 \pm 13 \mu \mathrm{m}$. $^{13}$

The gross images of MS/MgP composite scaffolds are shown in Figure 1A. The dimension of the MS/MgP scaffolds $(10 \times 10 \times 4 \mathrm{~mm})$ was well maintained without any expansion or shrinkage during the whole manufacturing process. Due to the advantage of the PED system, the structures could be accurately controlled so as to fabricate well-interconnected porous 3D scaffolds. A gradual increase in blue color could be seen while adding the blue-stained MSs into $\mathrm{MS} / \mathrm{MgP}$ composite scaffolds ranging from $0 \%$ to $20 \%(\mathrm{w} / \mathrm{w})$. This was more evident when observing the $\mathrm{MS} / \mathrm{MgP}$ composite scaffolds under a stereomicroscope (Figure 1B). Moreover, it seemed that the surface of the composite scaffolds became rougher as more MS content was added to MS/MgP scaffolds. The strut thickness and pore size of all $\mathrm{MS} / \mathrm{MgP}$ composite scaffolds were similar, at 540-580 $\mu \mathrm{m}$ and $420-440 \mu \mathrm{m}$, respectively $(n=5)$. In addition, the apparent porosity was found to be $50 \%-52 \%(n=5)$.

In the SEM micrographs (Figure 2), the existence of MS was clearly seen, either in the core or on the surface of the struts. As was shown in Figure 1, more MSs could be found in the MS/MgP composite scaffolds while increasing MS content from $2 \%$ to $20 \%(\mathrm{w} / \mathrm{w})$. As a result, the $20 \% \mathrm{MS} / \mathrm{MgP}$ scaffold exhibited the highest distribution of MS content, whereas others contained relatively few MSs.

\section{Water absorption}

The capacity of water absorption (\%) by the MS/MgP scaffolds was explained in Figure 3. Bare MgP scaffold showed the lowest water absorption, of $39 \%$, after soaking in DI water for 3 hours. However, the water uptake increased gradually with increasing MS content from $2 \%$ to $20 \%$. The $10 \%$ and $20 \% \mathrm{MS} / \mathrm{MgP}$ composite scaffolds showed a dramatic increase in water uptake, to $48 \%$ and $53 \%$, respectively.

According to our previous work on HA-containing composite MSs, ${ }^{13}$ the water content (\%) of the composite MSs varied from $64.5 \%$ to $81.1 \%$, depending on the HA content 

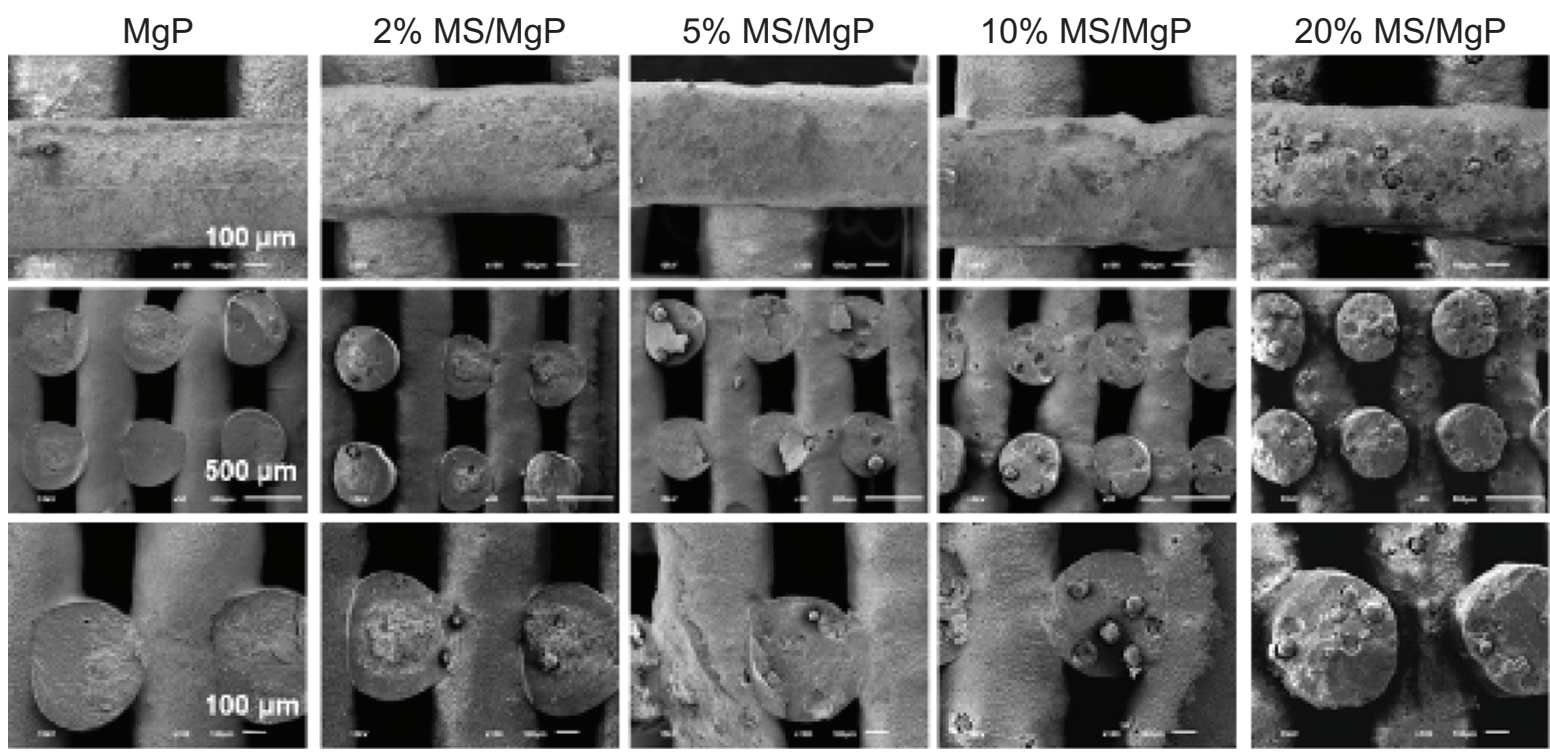

Figure 2 SEM measurement of MS/MgP composite scaffolds.

Notes: The surface (top row) and cross-sectional (middle and bottom row) micrographs were viewed at magnifications of $\times 50$ and $\times 100$.

Abbreviations: MgP, magnesium phosphate; MS, microsphere; SEM, scanning electron microscope.

embedded in polymeric MSs. In this work, the experiments were mostly conducted using 55\% HA-containing MSs. This MS has the lowest water content (\%), of 64.5\%, but still had excellent hydrophilic capacity. Due to the significant influence of MSs, it was possible to enhance the hydrophilic properties of bare $\mathrm{MgP}$ scaffold by simple incorporation of MS into the $\mathrm{MgP}$ matrix. Thus, $10 \%$ and $20 \% \mathrm{MS} / \mathrm{MgP}$ scaffolds were able to achieve improvement in water absorption (\%) relative to the $\mathrm{MgP}$ scaffold $(* * P<0.01)$. The proportional increase in water absorption $(\%)$ could be attributed to the

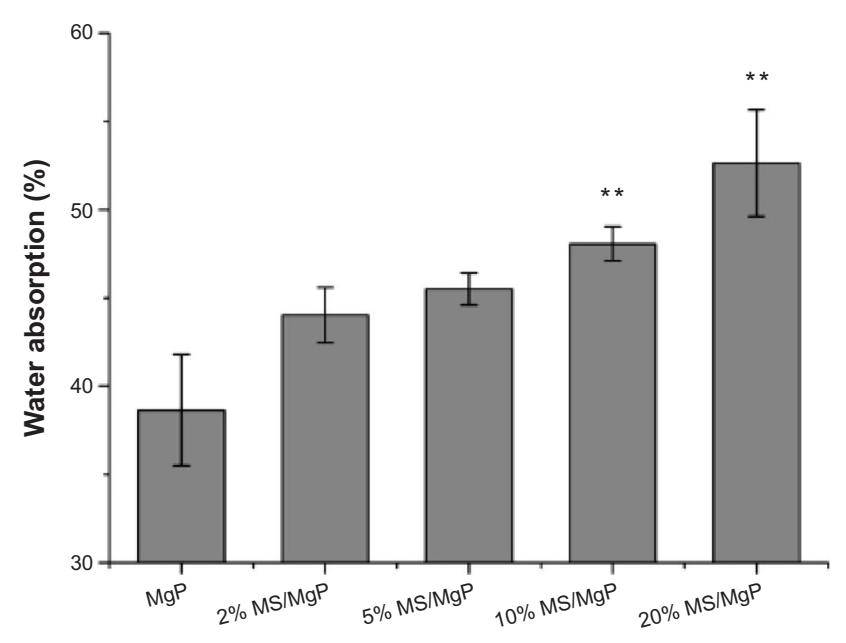

Figure 3 The water absorption (\%) of MS/MgP composite scaffolds was evaluated by comparing the weight differences between dried and hydrated samples.

Notes: MS ( $55 \%$ HA) was used to fabricate $0 \%$ to $20 \%$ MS/MgP composite scaffolds. A significant increase in water absorption (\%) was found in $10 \%$ and $20 \% \mathrm{MS} / \mathrm{MgP}$ scaffolds in comparison with MgP scaffolds $(* * P<0.01)$.

Abbreviations: $\mathrm{HA}$, hydroxyapatite; MgP, magnesium phosphate; MS, microsphere. increasing amount of hydrophilic composite MSs enveloped in the MS/MgP composite scaffolds.

\section{Compressive properties}

The compressive properties of $\mathrm{MS} / \mathrm{MgP}$ composite scaffolds are depicted in Figure 4. The compressive properties of $\mathrm{MgP}$ scaffolds are indicated using a dotted line for comparison. The HA-containing MS/MgP composite scaffolds maintained the compressive strength and modulus, both similar to the level of MgP scaffold throughout all MS content samples ( $2 \%$ to $20 \%$ ). However, $0 \%$ HA-containing MS/MgP scaffolds resulted in a gradual decrease in compressive strength, which finally reached its lowest, at 20\% MS/MgP scaffolds $(P<0.01)$. Regarding the compressive modulus, most of the $\mathrm{MS} / \mathrm{MgP}$ composite scaffolds exhibited similar values to the $\mathrm{MgP}$ scaffold, except for $20 \% \mathrm{MS} / \mathrm{MgP}$ scaffolds. In this case, a significant reduction was only seen in the $0 \%$ HA-containing $\mathrm{MS} / \mathrm{MgP}$ scaffold $(P<0.05)$. The inclusion of HA into MS was therefore advantageous to the MS/MgP composite scaffolds because the HA addition would be able to preserve the original mechanical characteristics of the MgP scaffold and also, prevent a substantial decrease in compressive properties.

\section{In vitro biodegradation and compressive properties}

The in vitro biodegradation of MS/MgP composite scaffolds were evaluated in two different aspects: the effect of $\mathrm{MS}$ content in MS/MgP scaffolds (Figure 5A) and of HA 
A

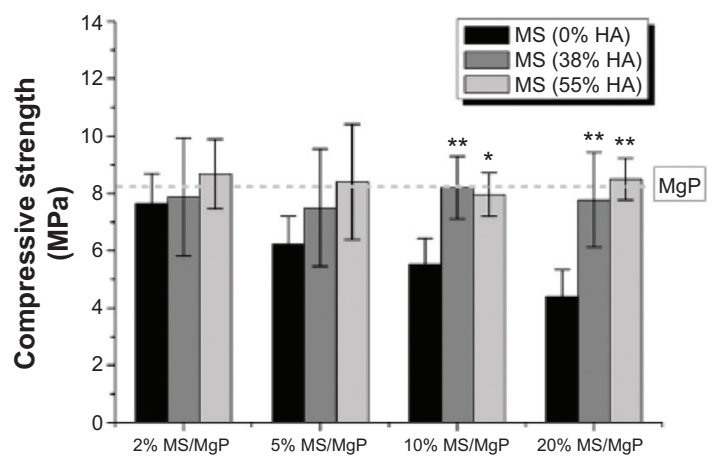

B

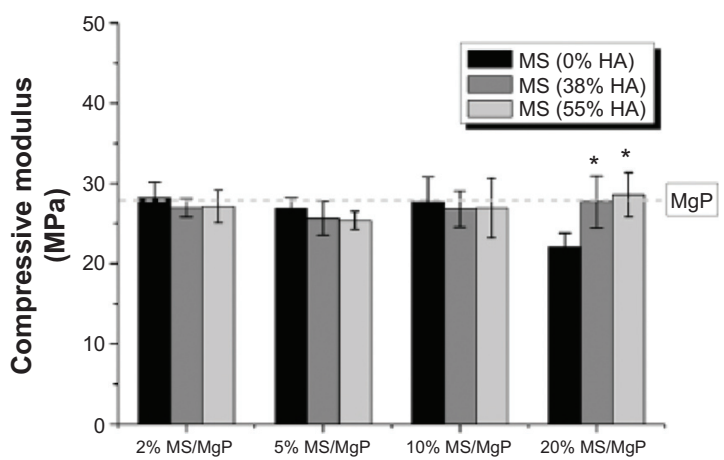

Figure 4 Compressive properties of MS/MgP composite scaffolds were tested to investigate the effect of MS content in MS/MgP scaffolds and of HA content in MS on compressive strength (A) and compressive modulus (B).

Notes: Statistical significance was evaluated in contrast to $0 \% \mathrm{HA}$-containing MS/MgP scaffolds at each group ( $* P<0.05$ or $* * P<0.01$ ).

Abbreviations: HA, hydroxyapatite; MgP, magnesium phosphate; MS, microsphere.

content in MS (Figure 5B). The steady increase in the in vitro biodegradation was monitored over time. This meant that all MS/MgP composite scaffolds would be degraded gradually in proportion to the incubation time. The in vitro biodegradation was facilitated with increasing MS content, from $0 \%$ to $20 \%$. The greatest weight reduction occurred with the $20 \% \mathrm{MS} /$ $\mathrm{MgP}$ composite scaffolds throughout the whole incubation time (day 5 and day $15 P<0.01$; day $30 P<0.05$ ). With regard to the influence of HA content in MS, a substantial increase in biodegradation (\%) was shown with the reduction of HA content from $55 \%$ to $0 \%(P<0.01)$.

The corresponding compressive properties at each biodegradation time are shown in Figure 6, and this revealed a close relationship to the in vitro biodegradation. The compressive strength of $\mathrm{MS} / \mathrm{MgP}$ composite scaffolds began to decrease after being biodegraded over time (Figure 6A). Contrary to the in vitro biodegradation result (Figure 5), the more the MS/MgP composite scaffolds were degraded, the fewer compressive properties were obtained as a function of biodegradation time. For this reason, the lowest compressive strength was found with $20 \% \mathrm{MS} / \mathrm{MgP}$ composite scaffolds from day 5 to day 30. Due to the superior biodegradability of the HA-containing $10 \% \mathrm{MS} / \mathrm{MgP}$ composite scaffolds, significant decrease in compressive properties was found with $0 \%$ and $38 \%$ HA-containing $10 \% \mathrm{MS} / \mathrm{MgP}$ scaffolds (Figure 6B). However, the 55\% HA-containing 10\% MS/ $\mathrm{MgP}$ scaffolds were able to prevent this significant loss of mechanical properties to some extent.

In Figure 5, the in vitro biodegradation pattern of MS/ $\mathrm{MgP}$ composite scaffolds was highly associated with MS
A

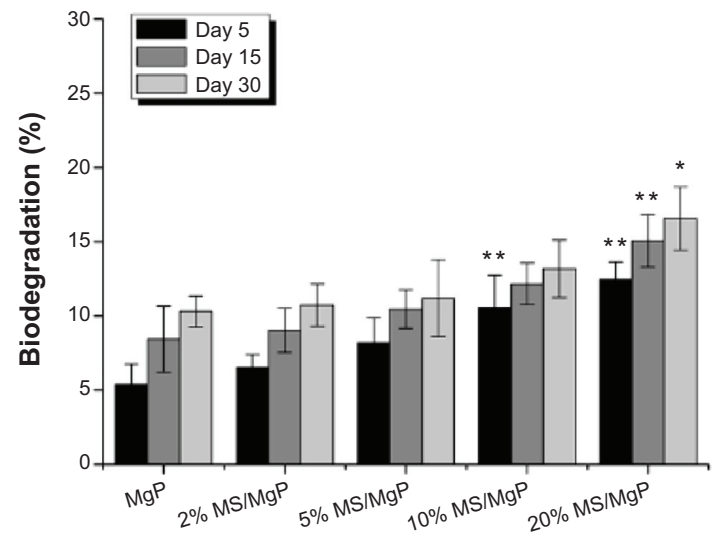

B

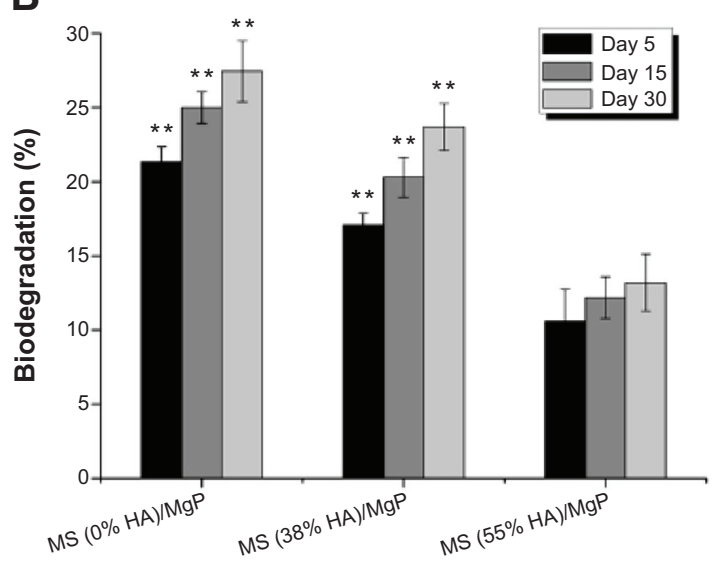

Figure 5 In vitro biodegradation of MS/MgP scaffolds was carried out by immersion in enzymatic solution for 30 days.

Notes: The effect of MS content in MS/MgP scaffolds (A) and of HA content in MS (B) was investigated. MS content was controlled from $0 \%$ to $20 \%$ in $55 \%$ HA-containing MS/MgP scaffolds; HA content was controlled from $0 \%$ to $55 \%$ in $10 \%$ MS/MgP scaffolds. Statistical significance was evaluated in contrast to MgP scaffolds (A) and MS (55\% $\mathrm{HA}) / \mathrm{MgP}$ scaffolds $(\mathbf{B})(* P<0.05$ or $* * P<0.0 \mathrm{I})$.

Abbreviations: $\mathrm{HA}$, hydroxyapatite; MgP, magnesium phosphate; MS, microsphere. 
A

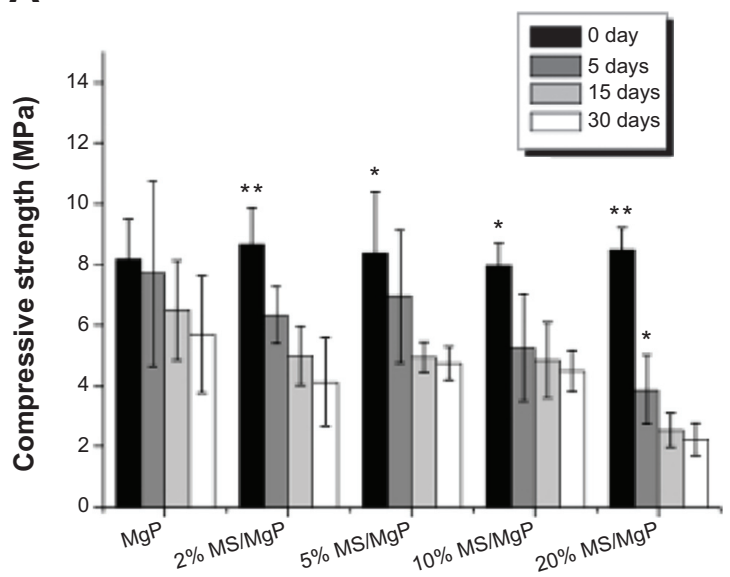

B

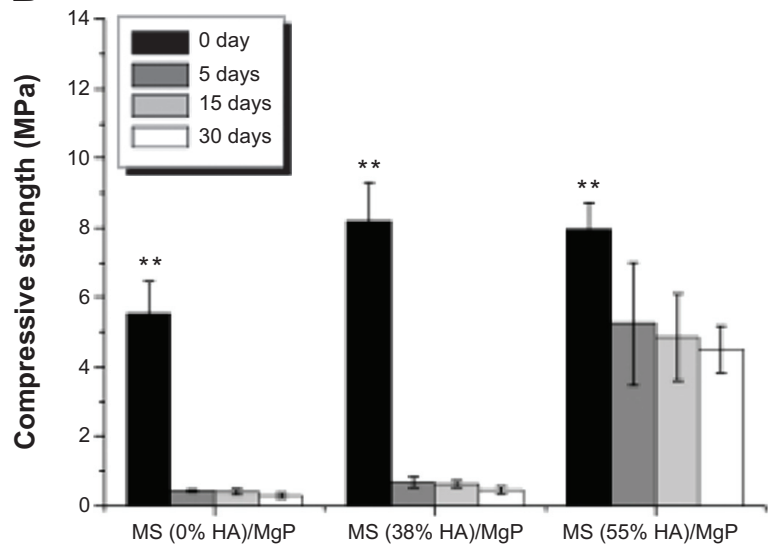

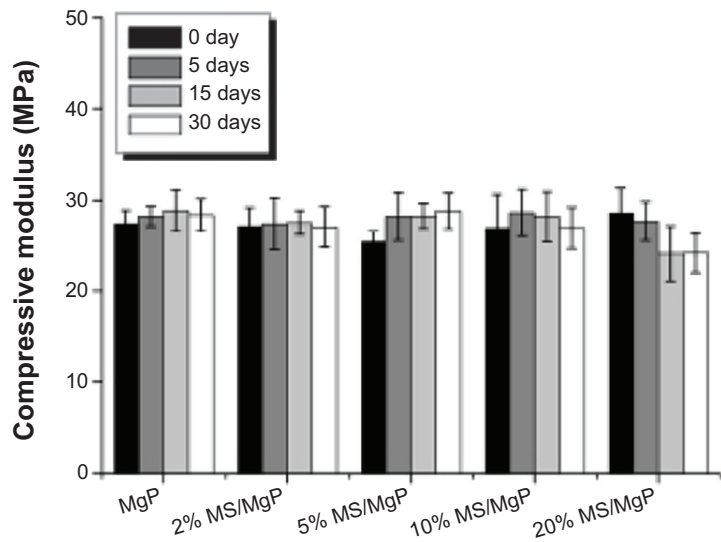

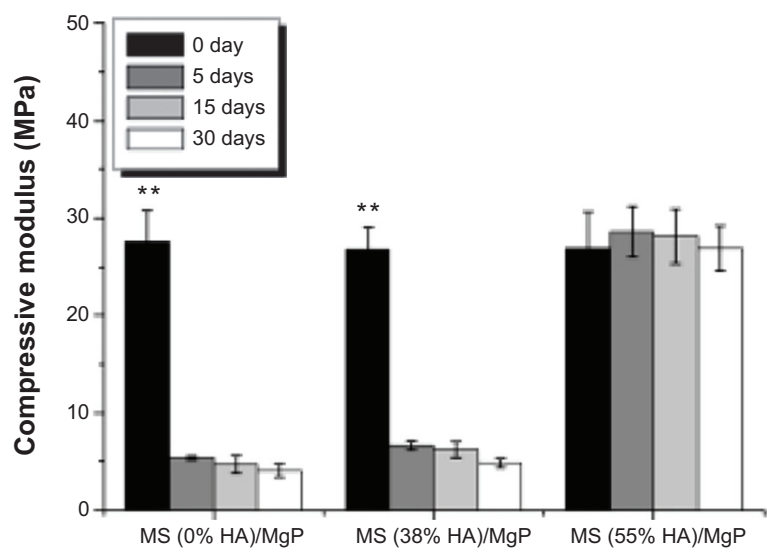

Figure 6 The corresponding compressive properties at each biodegradation time were evaluated with respect to compressive strength and modulus. Notes: The effect of MS content in MS/MgP scaffolds (A) and of HA content in MS (B) was investigated. MS content was controlled from $0 \%$ to $20 \%$ in $55 \%$ HA-containing $\mathrm{MS} / \mathrm{MgP}$ scaffolds; $\mathrm{HA}$ content was controlled from $0 \%$ to $55 \%$ in $10 \% \mathrm{MS} / \mathrm{MgP}$ scaffolds. Statistical significance was evaluated in contrast to scaffolds at day 30 ( $* \mathrm{P}<0.05$ or $* * \mathrm{P}<0.0 \mathrm{I})$.

Abbreviations: $\mathrm{HA}$, hydroxyapatite; MgP, magnesium phosphate; MS, microsphere.

content (\%), HA content (\%), and degradation time (day). In this work, we recognized that MS/MgP composite scaffolds would be susceptible to degrade in enzymatic solutions because the MSs were mainly constituted of biodegradable natural polymers, such as gelatin and chitosan. As a result, the largest weight reduction took place in the $20 \% \mathrm{MS} /$ $\mathrm{MgP}$ composite scaffolds at day 30 . The biodegradation of MS-embedding bioceramic scaffolds has been extensively studied under in vitro and in vivo circumstances. ${ }^{9,10}$ In most cases, the biodegradable polymeric MS was impregnated into bioceramic matrix in a certain ratio and kept under physiological conditions for a prolonged period of time. Then, the result was usually evaluated in terms of the morphology, porosity (\%), weight loss (\%), mechanical properties, and so on. For example, a composite scaffold of gelatin MS/ CPC was prepared to create a macroporous structure after being degraded in PBS for 5 weeks. ${ }^{15} \mathrm{~A}$ higher porosity was obtained with the degradation of gelatin MS. This could subsequently lead to a gradual decrease in compressive strength with MS content and degradation time, which is very consistent with our results as shown in Figures 5 and 6. As well, the bulk erosion of gelatin MSs began after 3 weeks degradation so that many macropores with interconnectivity was spread out everywhere.

Given the influence of HA content in MS, the inclusion of HA into the MSs was able to, not only retard the biodegradation process but also, compensate for the potential loss of compressive properties in a sustained manner. In comparison with the biodegradation (\%) at day 30,0\% HA-containing $10 \% \mathrm{MS} / \mathrm{MgP}$ scaffold was $27.5 \%$, which was twice as large as $13.2 \%$ (55\% HA-containing 10\% MS/ $\mathrm{MgP}$ scaffold; Figure 5B). However, the corresponding compressive strength of these two samples was measured as $0.3 \mathrm{MPa}$ and $4.5 \mathrm{MPa}$, respectively. It is therefore straightforward that the improved compressive properties of the $55 \%$ HA-containing $\mathrm{MS} / \mathrm{MgP}$ composite scaffolds were 
mostly attributed to the significant effect of HA inclusion. In order to reinforce the mechanical properties of bone tissue engineering scaffolds, HA has been typically incorporated into a bulk polymeric phase, with or without surface modification: PLLA, ${ }^{16}$ poly ( $\varepsilon$-caprolactone) $){ }^{17}$ and chitosan. ${ }^{18}$ In most cases, the compressive properties of HA-containing composite scaffolds would improve with the increasing weight ratio and remain stabilized until a certain point. This is mainly ascribed to the enhanced interaction between HA and the bulk matrix and good dispersion of HA inside the matrix. ${ }^{17}$ Furthermore, it was reported that the electrostatic interactions of gelatin and chitosan with HA nanoparticles could slow down the in vitro biodegradation and mechanical properties. ${ }^{18,19}$ As with the previous studies, we also confirmed the inverse relationship between the in vitro biodegradation and corresponding compressive properties of MS/MgP composite scaffolds. The critical importance of the HA addition into MSs was emphasized, so as to prevent a severe reduction of compressive properties with in vitro biodegradation time.

\section{In vitro lysozyme-release kinetics}

For this experiment, $10 \% \mathrm{MSs}$ with different HA content were homogeneously embedded into $\mathrm{MgP}$ scaffolds to investigate the effect of HA on the in vitro lysozymerelease kinetics. The in vitro release pattern was monitored for 30 days and analyzed in terms of cumulative release amount $(\mu \mathrm{g})$ (Figure 7A) and cumulative release percentage (\%) (Figure 7B). The lysozyme-loading content of MS and MS/MgP composite scaffolds could be obtained as follows. First, the lysozyme-loading content of $10 \%$
MS with different HA contents was: $810 \mu \mathrm{g}$ for MS ( $0 \% \mathrm{HA}), 889 \mu \mathrm{g}$ for MS (38\% HA), and $891 \mu \mathrm{g}$ for MS (55\% HA). Second, the final lysozyme-loading content of the $10 \% \mathrm{MS} / \mathrm{MgP}$ composite scaffolds was calculated based on the dried weight of each sample: $166 \mu \mathrm{g}$ for $\mathrm{MgP}$ scaffold, $109 \mu \mathrm{g}$ for MS ( $0 \% \mathrm{HA}$ )/MgP scaffold, 140 $\mu \mathrm{g}$ for MS (38\% HA)/MgP scaffold, and $140 \mu \mathrm{g}$ for MS (55\% HA)/MgP scaffold. In Figure 7A, the MgP scaffold, which had direct blending with lysozyme, showed an initial burst until 7 days and then continued to retain the highest release of lysozyme until 30 days. On the other hand, the MS/MgP composite scaffolds showed a controlled release rate without any serious loss and showed graded lysozyme-release patterns in accordance with HA content. The lysozyme release kinetics of composite scaffolds is shown in order of MS (38\% $\mathrm{HA}) / \mathrm{MgP}$ and $\mathrm{MS}$ $(55 \% \mathrm{HA}) / \mathrm{MgP}$. A slower release patten of MS $(55 \%$ $\mathrm{HA} / \mathrm{MgP}$ is attributed to the strong affinity of HA to lysozyme as depicted in Figure 7A.

In our previous study, an HA-containing gelatin/chitosan MS was developed as an efficient protein delivery carrier that not only enables the loading of high quantities of lysozyme, but also, maintains a sustained release without an initial burst. ${ }^{13}$ The HA effect on lysozyme delivery was enhanced with increases in HA content from $38 \%$ to $55 \%$. This outstanding feature could be explained by the increase in specific surface areas and strong electrostatic interactions of HA-containing composite MSs. For more advanced applications, we endeavored to combine the novel MS with $\mathrm{MgP}$ scaffolds, to allow for the higher loading capacity and prolonged release of lysozyme in a controlled manner. As
A

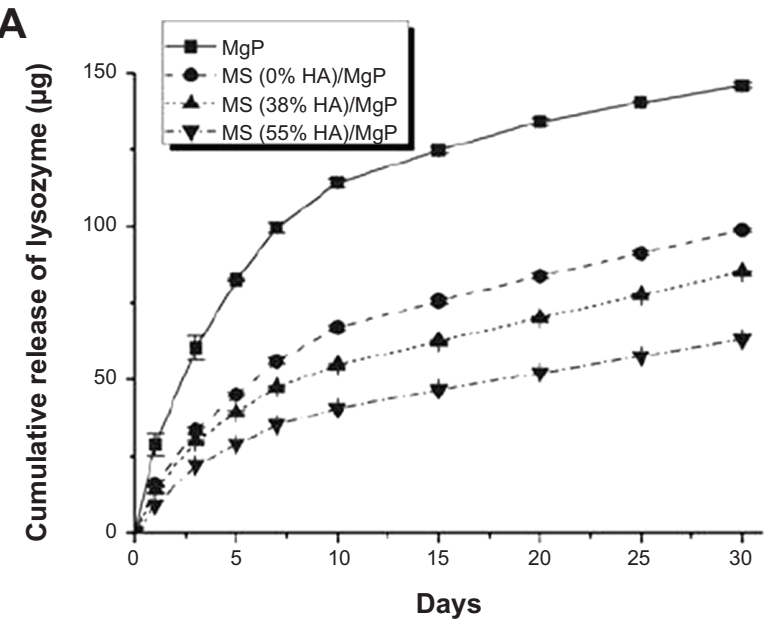

B

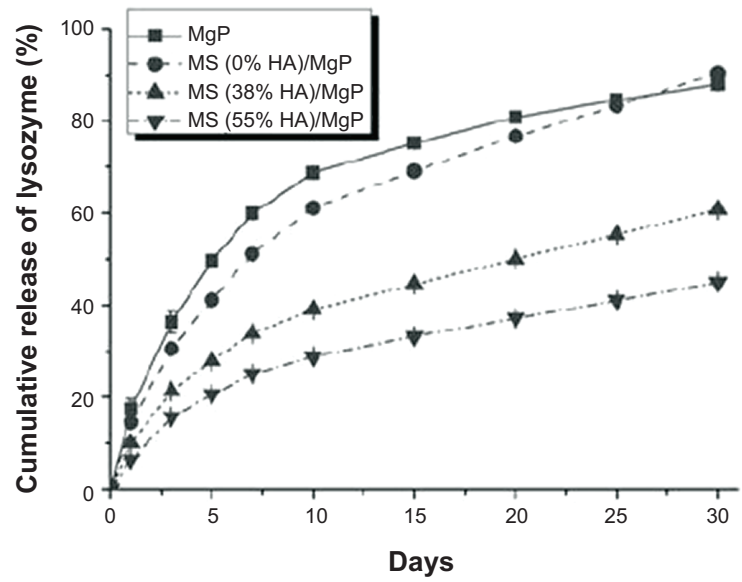

Figure 7 In vitro lysozyme-release kinetics of MS/MgP composite scaffolds was monitored for 30 days and expressed in two different aspects: (A) cumulative release amount $(\mu \mathrm{g})$ and $(\mathbf{B})$ cumulative release percentage (\%) against lysozyme loading amount. Abbreviations: HA, hydroxyapatite; MgP, magnesium phosphate; MS, microsphere. 
Table I Quantitative analysis of in vitro calcium deposition was carried out, in terms of MS content, in 55\% HA-containing MS/MgP scaffolds

\begin{tabular}{llllll}
\hline Ca deposition $(\mu \mathrm{g} / \mathrm{mL})$ & $\mathbf{M g P}$ & $\mathbf{2 \%} \mathbf{M S} / \mathbf{M g P}$ & $\mathbf{5 \%} \mathbf{M S} / \mathbf{M g P}$ & $\mathbf{1 0 \%} \mathbf{M S} / \mathbf{M g P}$ & $\mathbf{2 0 \%} \mathbf{M S} / \mathbf{M g P}$ \\
\hline Day 5 & $119 \pm 3$ & $120 \pm 2$ & $121 \pm 4$ & $126 \pm 6$ & $127 \pm 6$ \\
Day 10 & $229 \pm 4$ & $236 \pm 2 *$ & $239 \pm I^{* *}$ & $244 \pm 2^{* *}$ & $245 \pm I^{* *}$ \\
Day 20 & $34 I \pm 7$ & $354 \pm 3$ & $363 \pm 5 *$ & $372 \pm 4^{* *}$ & $376 \pm 4 * *$ \\
\hline
\end{tabular}

Notes: Values are expressed as the mean \pm standard deviation. Statistical significance was evaluated in contrast to MgP scaffolds at each time $(* P<0.05$ or $* * P<0.01)$.

Abbreviations: $\mathrm{HA}$, hydroxyapatite; MgP, magnesium phosphate; MS, microsphere.

presented in Figure 7, it was obvious that the incorporation of MS into MgP scaffold could result in a controllable lysozyme-release pattern without a severe initial burst. The slower release of lysozyme took place in the higher HAcontaining MS/MgP composite scaffolds; in contrast, the fast release of lysozyme was seen on the bare MgP scaffold, due to the rapid diffusion.

Various types of MSs have been incorporated into a bulk matrix consisting of either biodegradable polymers or bioceramics. ${ }^{20,21}$ In most cases, the MS-containing composite structures were usually aimed to overcome the conventional limitations of tissue engineering scaffolds, especially poor drug delivery ability. Based on recent publications, it would be feasible to achieve a sustained release of a single biomolecule or sequential delivery of dual bioactive molecules for the tissue engineering application. ${ }^{3,22}$ In view of clinical applications, antibiotics (eg, gentamicin) or growth factors (eg, bone-morphogenetic protein-2) were encapsulated within poly(lactic-co-glycolic acid) MSs and subsequently mixed with calcium phosphate bone cement to generate a controlled drug delivery system for the effective treatment of bone infections and bone regeneration, respectively. ${ }^{11,23}$

\section{In vitro calcification}

The ability of MS/MgP composite scaffolds to initiate in vitro calcification by SBF treatment was evaluated in two different aspects: the effect of MS content in MS/MgP scaffolds (Table 1) and of HA content in MS (Table 2). MgP scaffolds contained the lowest calcium ion deposition throughout the entire incubation time. On the other hand, MS/MgP composite scaffolds steadily enhanced the in vitro calcium deposition, while increasing MS content from $2 \%$ to $20 \%$. At day 20 , the $10 \%$ and $20 \% \mathrm{MS} / \mathrm{MgP}$ composite scaffolds resulted in the highest quantity of calcium ion deposition $(P<0.01)$ (Table 1). With respect to HA content in MS, $55 \%$ HA-containing MS/MgP composite scaffolds led to the highest level of calcium ion deposition $(P<0.05)$, followed by $38 \%$ HA-containing MS/MgP scaffolds (Table 2 ).
In the SEM morphological analysis (Figure 8), the flakelike apatite crystals were newly produced and distributed on the surface of all MS/MgP composite scaffolds. As with the quantitative result of calcium ion deposition (Table 1), 20\% $\mathrm{MS} / \mathrm{MgP}$ composite scaffolds could generate relatively more apatite crystals than other MS/MgP scaffolds (Figure 8A). Nevertheless, it was difficult to find morphological variations between the different HA-containing $\mathrm{MS} / \mathrm{MgP}$ scaffolds (Figure 8B). The elemental analysis of the neoformation of apatite crystals was also carried out by means of EDS. A representative EDS result is illustrated, in Figure 8A, to confirm the noticeable peaks for calcium and phosphate. The EDS patterns were comparable with each other, regardless of different MS and HA content.

The effect of HA addition on in vitro calcification of HA-containing MSs was already investigated in our previous study, by soaking them in SBF at physiological conditions. ${ }^{13}$ We found that the calcium ion deposition was facilitated with increasing incubation time and HA content in MSs. This result is consistent with the present work using MS/ $\mathrm{MgP}$ composite scaffolds (Tables 1 and 2). The in vitro calcium deposition was proportional to MS content in MS/ $\mathrm{MgP}$ scaffold, HA content in MSs, and the incubation time. Overall, the important role of nanosized HA would be able to accelerate the in vitro apatite-forming ability of $\mathrm{MS} / \mathrm{MgP}$ composite scaffolds by SBF treatment. Using morphological and elemental analysis, flake-like apatite crystals and noticeable calcium/phosphate peaks were obviously detected in all SBF-treated scaffolds, respectively (Figure 8).

Table 2 Quantitative analysis of in vitro calcium deposition was carried out, in terms of HA content, in $10 \% \mathrm{MS} / \mathrm{MgP}$ scaffolds

\begin{tabular}{llll}
\hline Ca deposition & MS (0\% HA)/ & MS (38\% HA)/ & MS (55\% HA)/ \\
$(\mu \mathrm{g} / \mathrm{mL})$ & MgP & MgP & MgP \\
\hline Day 5 & $124 \pm 2$ & $123 \pm 1$ & $126 \pm 6$ \\
Day 10 & $234 \pm 4$ & $240 \pm 1$ & $244 \pm 2 *$ \\
Day 20 & $350 \pm 4$ & $355 \pm 2$ & $372 \pm 4 * *$ \\
\hline
\end{tabular}

Notes: Values are expressed as the mean \pm standard deviation. Statistical significance was evaluated in contrast to MS $(0 \% \mathrm{HA}) / \mathrm{MgP}$ scaffolds at each time $(* P<0.05$ or $* *$ P $<0.0$ l).

Abbreviations: HA, hydroxyapatite; MgP, magnesium phosphate; MS, microsphere. 

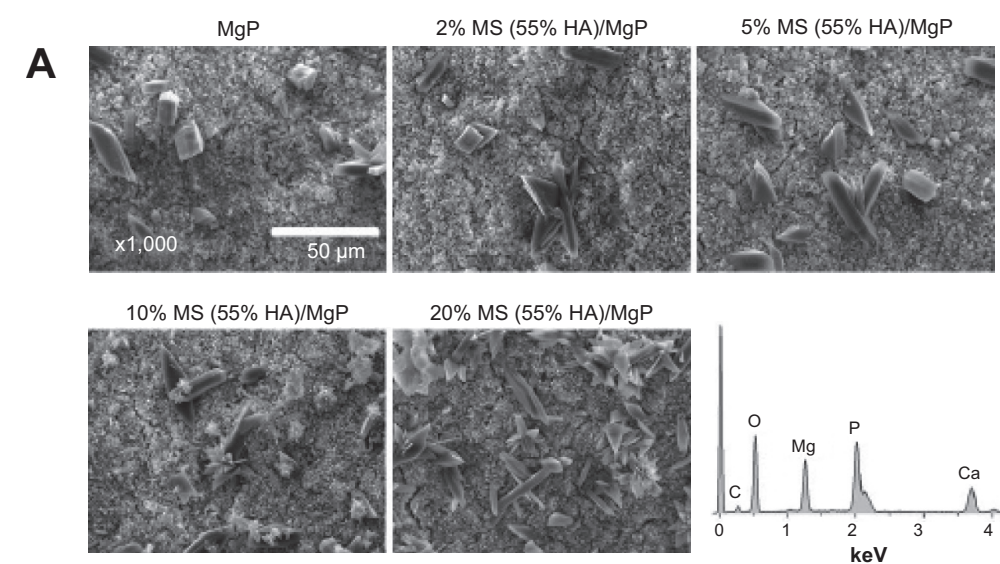

$20 \% \mathrm{MS}(55 \% \mathrm{HA}) / \mathrm{MgP}$
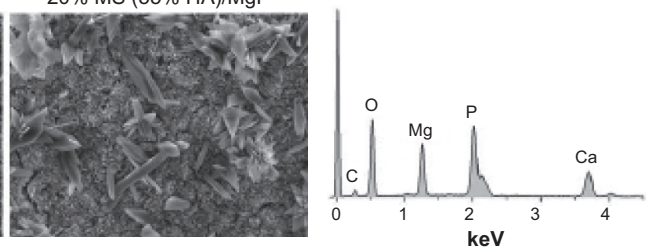

B
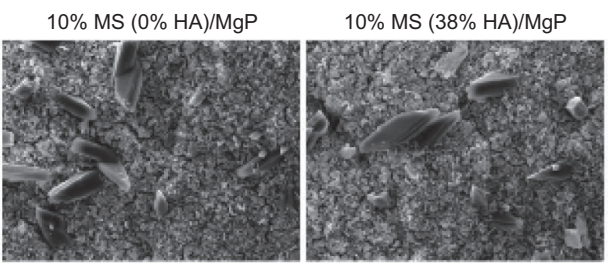

$10 \% \mathrm{MS}(55 \% \mathrm{HA}) / \mathrm{MgP}$

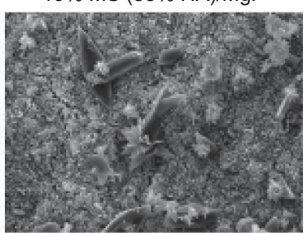

Figure 8 SEM measurement of MS/MgP scaffold after SBF treatment for 20 days ( $\times 1,000$ magnification).

Notes: Newly-formed apatite crystals were observed regardless of (A) MS content in MS (55\% HA)/MgP scaffolds and (B) HA content in I0\% MS. (A) The EDS result was similar (right bottom).

Abbreviations: EDS, energy dispersive spectroscopy; HA, hydroxyapatite; MgP, magnesium phosphate; MS, microsphere; SBF, simulated body fluid; SEM, scanning electron microscope.

However, there was no evidence of apatite formation in bare $\mathrm{MgP}$ scaffold kept in DI water for the same period of time (Figure S1). To date, SBF solution has been widely utilized for the functionalization of biomaterials as it could mimic the actual physiological conditions and consequently, improve both in vitro bioactivity and in vivo performance. To this end, many types of biomaterials would be able to create the bone-like apatite layers on the surface and integrate with surrounding host bony tissues, without forming fibrous interfacial tissue. ${ }^{24}$

\section{Conclusion}

A synergistic combination of MgP scaffold and MS was carried out in this work to fulfill the requirements for bone tissue engineering scaffold. Porous 3D structures with good interconnectivity were fabricated using a PED system. Controlled lysozyme-release kinetics were exhibited in accordance with HA content. In vitro biodegradation was directly affected by MS and HA content throughout the entire incubation period, which led to an inverse relationship with corresponding compressive properties. In vitro calcium deposition was initiated by SBF treatment so that the biomimetic apatite crystals could be formed on the MS/MgP composite scaffolds. Taken together, these results suggest that an MS/MgP composite scaffold is a promising candidate material to achieve sustained drug-release capacity, biodegradability, and bioactivity for effective bone regeneration.

\section{Acknowledgments}

This work was supported by the Mid-Career Researcher Program, through a National Research Foundation of Korea (NRF) grant funded by the Korea Ministry of Education, Science, and Technology (MEST) (number 2011-0017572).

\section{Disclosure}

The authors report no conflicts of interest in this work.

\section{References}

1. Wang H, Leeuwenburgh SC, Li Y, Jansen JA. The use of micro- and nanospheres as functional components for bone tissue regeneration. Tissue Eng Part B Rev. 2012;18(1):24-39.

2. Habraken WJ, Wolke JG, Mikos AG, Jansen JA. Injectable PLGA microsphere/calcium phosphate cements: physical properties and degradation characteristics. J Biomater Sci Polym Ed. 2006;17(9):1057-1074.

3. Niu X, Feng Q, Wang M, Guo X, Zheng Q. Porous nano-HA/collagen/ PLLA scaffold containing chitosan microspheres for controlled delivery of synthetic peptide derived from BMP-2. J Control Release. 2009; 134(2):111-117.

4. Park H, Temenoff JS, Holland TA, Tabata Y, Mikos AG. Delivery of TGF-beta1 and chondrocytes via injectable, biodegradable hydrogels for cartilage tissue engineering applications. Biomaterials. 2005;26(34): 7095-7103.

5. Zhu G, Mallery SR, Schwendeman SP. Stabilization of proteins encapsulated in injectable poly(lactide-co-glycolide). Nat Biotechnol. 2000; 18(1):52-57. 
6. Kempen DH, Kruyt MC, Lu L, et al. Effect of autologous bone marrow stromal cell seeding and bone morphogenetic protein-2 delivery on ectopic bone formation in a microsphere/poly(propylene fumarate) composite. Tissue Eng Part A. 2009;15(3):587-594.

7. Matsuno T, Hashimoto Y, Adachi S, et al. Preparation of injectable 3D-formed beta-tricalcium phosphate bead/alginate composite for bone tissue engineering. Dent Mater J. 2008;27(6):827-834.

8. Wang C, Gong Y, Zhong Y, Yao Y, Su K, Wang DA. The control of anchorage-dependent cell behavior within a hydrogel/microcarrier system in an osteogenic model. Biomaterials. 2009;30(12):2259-2269.

9. Habraken WJ, Liao HB, Zhang Z, et al. In vivo degradation of calcium phosphate cement incorporated into biodegradable microspheres. Acta Biomater. 2010;6(6):2200-2211.

10. Félix Lanao RP, Leeuwenburgh SC, Wolke JG, Jansen JA. In vitro degradation rate of apatitic calcium phosphate cement with incorporated PLGA microspheres. Acta Biomater. 2011;7(9):3459-3468.

11. Schnieders J, Gbureck U, Thull R, Kissel T. Controlled release of gentamicin from calcium phosphate-poly(lactic acid-co-glycolic acid) composite bone cement. Biomaterials. 2006;27(23):4239-4249.

12. Lee J, Farag MM, Park EK, Lim J, Yun HS. A simultaneous process of 3D magnesium phosphate scaffold fabrication and bioactive substance loading for hard tissue regeneration. Mater Sci Eng C Mater Biol Appl. 2014;36:252-260.

13. Lee J, Yun HS. Hydroxyapatite-containing gelatin/chitosan microspheres for controlled release of lysozyme and enhanced cytocompatibility. J Mater Chem B. 2014;2(9):1255-1263.

14. Kokubo T, Takadama H. How useful is SBF in predicting in vivo bone bioactivity? Biomaterials. 2006;27(15):2907-2915.

15. Li M, Liu X, Liu X, Ge B, Chen K. Creation of macroporous calcium phosphate cements as bone substitutes by using genipin-crosslinked gelatin microspheres. J Mater Sci Mater Med. 2009;20(4):925-934.
16. Fang Z, Feng Q. Improved mechanical properties of hydroxyapatite whisker-reinforced poly(L-lactic acid) scaffold by surface modification of hydroxyapatite. Mater Sci Eng C Mater Biol Appl. 2014;35:190-194.

17. Wang Y, Dai J, Zhang Q, Xiao Y, Lang M. Improved mechanical properties of hydroxyapatite/poly( $\varepsilon$-caprolactone) scaffolds by surface modification of hydroxyapatite. Appl Surf Sci. 2010;256(20):6107-6112.

18. Thein-Han WW, Misra RD. Biomimetic chitosan-nanohydroxyapatite composite scaffolds for bone tissue engineering. Acta Biomater. 2009; 5(4):1182-1197.

19. Leeuwenburgh SC, Jo J, Wang H, Yamamoto M, Jansen JA, Tabata Y. Mineralization, biodegradation, and drug release behavior of gelatin/ apatite composite microspheres for bone regeneration. Biomacromolecules. 2010;11(10):2653-2659.

20. Tang G, Zhang H, Zhao Y, Li X, Yuan X, Wang M. Prolonged release from PLGA/HAp scaffolds containing drug-loaded PLGA/gelatin composite microspheres. J Mater Sci Mater Med. 2012;23(2):419-429.

21. Girod Fullana S, Ternet H, Freche M, Lacout JL, Rodriguez F. Controlled release properties and final macroporosity of a pectin microspheres-calcium phosphate composite bone cement. Acta Biomater. 2010;6(6):2294-2300.

22. Wang X, Wenk E, Zhang X, Meinel L, Vunjak-Novakovic G, Kaplan DL. Growth factor gradients via microsphere delivery in biopolymer scaffolds for osteochondral tissue engineering. J Control Release. 2009;134(2):81-90.

23. Ruhé PQ, Boerman OC, Russel FG, Spauwen PH, Mikos AG, Jansen JA. Controlled release of rhBMP-2 loaded poly(dl-lactic-co-glycolic acid)/ calcium phosphate cement composites in vivo. $J$ Control Release. 2005;106(1-2):162-171.

24. Zadpoor AA. Relationship between in vitro apatite-forming ability measured using simulated body fluid and in vivo bioactivity of biomaterials. Mater Sci Eng C Mater Biol Appl. 2014;35:134-143. 


\section{Supplementary material}
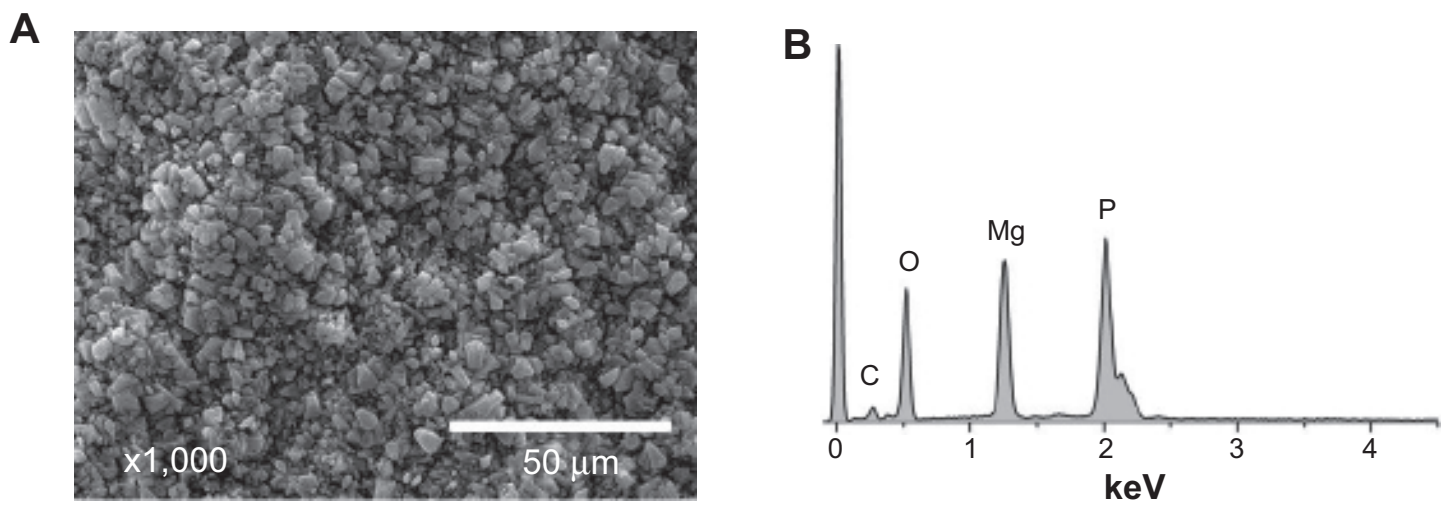

Figure SI SEM measurement of the MgP scaffold incubated in DI water for 20 days $(\times \mathrm{I}, 000$ magnification) $(\mathbf{A})$ and the corresponding EDS result $(\mathbf{B})$ were presented as a negative control for SBF treatment.

Note: No apatite crystals or noticeable Ca peak were detected.

Abbreviations: DI, deionized; EDS, energy dispersive spectroscopy; MgP, magnesium phosphate; SBF, simulated body fluids; SEM, scanning electron microscope.

\section{Publish your work in this journal}

The International Journal of Nanomedicine is an international, peerreviewed journal focusing on the application of nanotechnology in diagnostics, therapeutics, and drug delivery systems throughou the biomedical field. This journal is indexed on PubMed Central, MedLine, CAS, SciSearch $®$, Current Contents $\AA /$ Clinical Medicine,
Journal Citation Reports/Science Edition, EMBase, Scopus and the Elsevier Bibliographic databases. The manuscript management system is completely online and includes a very quick and fair peer-review system, which is all easy to use. Visit http://www.dovepress.com/ testimonials.php to read real quotes from published authors. 